\title{
EFEITO DE DIFERENTES DOSES DE ENXOFRE NO CONSUMO VOLUNTÁRIO E NAS POPULAĈ̃ES DE PROTOZOÁRIOS DO RÚMEN DE NOVILHAS MESTIÇAS ALIMENTADAS COM CAPIM-ELEFANTE DEE BAIXA QUALIDADE
}

\author{
Effect of different doses of sulfur on voluntary intake of low-quality elephant grass and \\ estimates of ruminal protozoa populations in crossbred heifers
}

\author{
Juliana Miacci Vidal ${ }^{1}$, Paulo César de Aguiar Paiva², Pedro Braga Arcuri ${ }^{3}$, \\ Fernando César Ferraz Lopes $^{4}$, Leonardo Zaquini ${ }^{5}$
}

\begin{abstract}
RESUMO
O experimento foi conduzido no Campo Experimental de Coronel Pacheco-MG da EMBRAPA Gado de Leite. O efeito de doses de enxofre (sulfato de amônio, 0,$15 ; 0,31 ; 0,46$ e $0,92 \%$ de S na matéria verde / dia) na população de protozoários ruminais, foi avaliado utilizando-se quatro novilhas $7 / 8$ Holandês X Zebu, arranjadas em um quadrado latino de 4 x 4 . Forneceu-se diariamente capim-elefante de baixa qualidade (76,1\% FDN na MS), picado, com correção do teor de PB para 7\% com uréia, mais mistura mineral sem enxofre fornecida diretamente no rúmen. Foram feitas amostragens do conteúdo ruminal, uma hora após a alimentação. A estimativa das populações microbianas ruminais foi realizada por microscópica direta. Os resultados foram transformados para logaritmos decimais e avaliados estatisticamente. Não houve diferença significativa entre os tratamentos para microrganismos. $\mathrm{O}$ tratamento $0,92 \% \mathrm{~S}$ apresentou o menor consumo de matéria seca e ainda, causou início de intoxicação em dois animais. De acordo com esses resultados, doses de $0,31 \%$ de enxofre adicionadas a dieta promoveram o incremento das populações de microganismos ruminais e com isso um maior consumo voluntário.
\end{abstract}

Termos de indexação: Enxofre, protozoários ruminais, consumo voluntário.

\begin{abstract}
The experiment was carried out in the Experimental Field of Coronel Pacheco belonging to EMBRAPA Dairy Cattle, Minas Gerais. The effects of the doses of sulfur (ammonium sulfate, $0.15 \%, 0.31 \%, 0.46 \%$ and $0.92 \% \mathrm{~S}$ fresh matter/day) on the ruminal protozoa population was evaluated by utilizing four $7 / 8$ Holstein $\mathrm{x}$ Zebu, heifers arranged in $4 \mathrm{x} 4$ Latin square. Low quality elephant grass (76.1\% NDF in DM), chopped with correction of the CP content to 7\% with urea plus a mineral mixture without sulfur given directly into the rumen. Samplings of the ruminal content were done, one hour after feeding. The estimate of the ruminal microbial populations was done by means of the direct microscopy. The results were transformed to decimal logarithm and evaluated statistically. There were no significant differences among the treatments for microorganisms. The $0.92 \% \mathrm{~S}$ treatment presented the least dry matter intake and, in addition, caused start of intoxication in two animals. According to those results, doses of $0.31 \%$ of sulfur added to the diet promoted the increase of the ruminal microorganism populations and hence a greater voluntary intake.
\end{abstract}

Index terms: Sulfur, ruminal protozoa, voluntary intake.

(Recebido para publicação em 19 de abril de 2005 e aprovado em 27 de dezembro de 2005)

\section{INTRODUÇÃo}

A microbiota ruminal é composta, principalmente, por três grupos de microrganismos anaeróbios, as bactérias, os protozoários e os fungos. As populações de cada grupo e as espécies componentes destas são influenciadas diretamente pelo tipo de alimento fornecido ao hospedeiro. Em animais submetidos a dietas de baixo valor nutritivo e ricas em fibras, observa-se o aumento no número de protozoários ciliados e de fungos.

Os protozoários são microrganismos unicelulares, anaeróbios, não patogênicos, que variam em tamanho de
20 a $200 \mathrm{~mm}$ (portanto, cerca de 10 a 100 vezes maiores que as bactérias) (DEHORITY, 1993). Apresentam uma organização interna, com estruturas funcionais semelhantes a boca, o esôfago, o estômago, o reto, o ânus. Em algumas espécies, ocorre ainda uma placa rígida, semelhante a um esqueleto (DEHORITY, 1993). A população de protozoários do conteúdo ruminal de animais alimentados com diferentes tipos de dieta varia em concentração, entre $10^{4}$ e $10^{6}$ protozoários/ml de conteúdo ruminal (D’AGOSTO et al., 2001). Devido ao seu tamanho, essa concentração em geral representa de 40 a $60 \%$ da biomassa microbiana total do rúmen (EZEQUIEL, 2002). Como consequência, a presença

\footnotetext{
${ }^{1}$ Mestre em Zootecnia, com concentração em Nutrição de Ruminantes pela Universidade Federal de Lavras/UFLA - Cx. P 3037 - $37200-000$ Lavras, MG - miaccividal@yahoo.com.br

2 Professor titular do Departamento de Zootecnia da Universidade Federal de Lavras/UFLA - Cx. P 3037 - $37200-000$ - Lavras, MG.

${ }^{3}$ Pesquisador da EMBRAPA Gado de Leite - Rua Eugênio do Nascimento, 610 - Dom Bosco - 30.038-330 - Juiz de Fora, MG.

${ }^{4}$ Pesquisador da EMBRAPA Gado de Leite - Rua Eugênio do Nascimento, 610 - Dom Bosco - 30.038-330 - Juiz de Fora, MG.

${ }^{5}$ Graduando em Ciências Biológicas da Universidade Federal Juiz de Fora/UFJF - Campus Universitário - 36.036-330 - Juiz de Fora, MG.
} 
de protozoários no rúmen traz efeitos benéficos e outros adversos para a produção animal. Alguns protozoários são celulolíticos, mas os principais substratos utilizados pela fauna ruminal como fonte de energia são os açúcares e amidos, que são assimilados rapidamente e estocados na forma de amilopectina ou amido protozoário (WILLIAMS, 1986). Protozoários têm seu requerimento nutricional pouco estudado, pois das muitas espécies conhecidas, apenas cerca de 20 já foram cultivadas in vitro com sucesso (WILLIAMS \& COLEMAN, 1997). Entretanto, ao revisar este tópico, Williams \& Coleman (1997) sugerem que os requerimentos nutricionais sejam muito semelhantes aos de bactérias.

O enxofre é parte da predição das exigências de componentes orgânicos específicos e das exigências da microbiota ruminal (ANDRIGUETTO, 1993). A quantidade inadequada deste nas dietas pode prejudicar o metabolismo da microbiota ruminal, conduzindo à diminuição na digestão ruminal de carboidratos dietéticos e à diminuição no suprimento de aminoácidos para o metabolismo dos tecidos do hospedeiro. Tais mudanças causam a redução no consumo voluntário, no suprimento de energia e na taxa de síntese da proteína corporal (WESTON et al., 1988).

Quando um feno de gramínea tropical que possuía um teor de enxofre de $0,04 \%$ de MS, foi suplementado para 0,07\% de MS, observou-se o aumento de bactérias, protozoários e fungos anaeróbicos no rúmen (MORRISON et al., 1990).

Objetivou-se, com este trabalho, estudar o efeito de diferentes níveis de enxofre na dieta de bovinos leiteiros canulados, submetidos à dieta de baixo valor nutritivo e elevado teor de fibras, relacionando o consumo voluntário com a população de protozoários ruminais.

\section{MATERIALE MÉTODOS}

O experimento foi conduzido no período de junho a novembro de 2003, no Campo Experimental de Coronel Pacheco (CECP), pertencente à EMBRAPA Gado de Leite. Foram utilizadas quatro novilhas 7/8 Holandês x Zebu, não gestantes, não lactantes, com peso médio de $450 \mathrm{Kg}$, fistuladas no rúmen e providas de cânulas de látex permanentes. Os animais permaneceram em baias individuais com aproximadamente $50 \mathrm{~m}^{2}$, recebendo alimento à vontade e fornecido uma vez ao dia, além de livre acesso à água.

Foram avaliados quatro níveis de $\mathrm{S}$ definidos a partir da recomendação do NRC (2001) para o S total da dieta dos bovinos leiteiros de $0,32 \%$ da matéria seca ingerida por dia, conforme os tratamentos a seguir:

- Tratamento $0,15 \%$ de $\mathrm{S}$ na dieta

- Tratamento $0,31 \%$ de $\mathrm{S}$ na dieta

- Tratamento $0,46 \%$ de $\mathrm{S}$ na dieta

- Tratamento $0,92 \%$ de S na dieta

O enxofre foi fornecido na forma de sulfato de amônio e o cálculo da quantidade tomou como base o consumo médio de MS verificado na semana anterior. Nos tratamentos, foram consideradas as quantidades de $\mathrm{S}$ verificadas na semana anterior mais o $\mathrm{S}$ contido na forragem. Adicionou-se uréia ao capim picado, a fim de que todas as dietas apresentassem $7 \%$ de proteína bruta na matéria seca (NRC, 2001). A mistura mineral fornecida foi preparada pela NUTRIPLAN Ltda., de Juiz de Fora, Minas Gerais de modo a não conter $\mathrm{S}$.

O sulfato de amônio e o sal mineralizado foram pesados diariamente e individualmente em sacos de papel de 5 x $10 \mathrm{~cm}$ e imersos no conteúdo ruminal pela cânula, permitindo a mistura rápida no rúmen. Os animais receberam, diariamente, capim-elefante picado e maduro, com aproximadamente 120 dias de idade.

Os animais foram submetidos aos tratamentos num arranjo em quadrado latino 4 x 4 (quatro animais submetidos aos quatro tratamentos). Cada período experimental teve a duração de 21 dias, sendo 7 dias de adaptação e 14 dias de coletas.

O modelo estatístico adotado na análise de variância das médias dos dados está descrito abaixo:

$$
Y i j k l=\mu+\theta i+D(i) j+P(i) k+\pi l+\pi \theta i l+e i j k l
$$

sendo:

$\mathrm{Y}$ ijkl = valor para as variáveis consumo de capim-elefante e contagem microbiana

$\mathrm{m}=$ média geral

$\mathrm{q} i \mathrm{i}=$ efeito do quadrado latino i $(\mathrm{i}=1,2,3$ e 4$)$

$\mathrm{D}(\mathrm{i}) \mathrm{j}=$ efeito do animal $\mathrm{j}(\mathrm{j}=1,2,3$ e 4$)$

$\mathrm{P}(\mathrm{i}) \mathrm{k}=$ efeito do período $\mathrm{k}(\mathrm{k}=1,2,3$ e 4$)$

p 1 = efeito do tratamento $1(1=1,2,3$ e 4$)$

p q il = efeito da interação do quadrado i x tratamento 1

eijkl = erro associado a todas observações

Os dados de consumo de capim-elefante e número de protozoários foram submetidos à análise de variância e ao teste de Tuckey, a 5\% de significância (SAEG, 2000).

A coleta do conteúdo ruminal foi feita sempre uma hora após a alimentação. O conteúdo ruminal foi amostrado colhendo-se manualmente alíquotas de posições 
diferentes do rúmen, sendo estas imediatamente colocadas em garrafas térmicas devidamente identificadas, desinfetadas e pré-aquecidas com água a $40^{\circ} \mathrm{C}$.

O procedimento para as contagens de protozoários é realizado conforme descrito por D'Agosto \& Carneiro (1999).

Os valores de contagem de protozoários totais foram transformados para $\log _{10}(\mathrm{x}+1)$, conforme recomendação de Obispo \& Dehority (1992).

As análises das amostras da dieta (forragem + uréia + sulfato de amônio), do conteúdo ruminal (digesta) e das fezes, quanto aos teores de matéria seca (MS), proteína bruta (PB), foram realizadas de acordo com as metodologias descritas em AOAC (1990). As fibras em detergente neutro (FDN) e detergente ácido (FDA) foram determinadas de acordo com as metodologias descritas por Silva (1990).

\section{RESULTADOS E DISCUSSÃO}

Como pode ser verificado nos dados da Tabela 1, houve uniformidade na composição bromatológica do capim durante todo experimento. $\mathrm{O}$ capim utilizado tinha aproximadamente 120 dias, sem adubação recente. Os resultados encontrados estão de acordo com os encontrados por Gomes et al. (1994), que trabalharam com feno de capim-cameroom com altura de corte de $3 \mathrm{~m}$, apresentando 74,4\% de FDN e 49,0\% de FDA. Vieira et al. (1997), trabalhando com idades diferentes de capimelefante, encontraram no capim com 124 dias, teores MS de $26,2 \%$, FDN de $69,7 \%$ e FDA de 52,5\%.

A proteína bruta do experimento foi controlada de acordo com cada tratamento com o uso de uréia para ser utilizado o teor de 7\%, conforme recomendado pelo NRC (2001).

O consumo de MS pelos animais (Tabela 2) manteve-se estável nos tratamentos $0,15 \%, 0,31 \%$ e $0,46 \%$, não ocorrendo diferença significativa entre os mesmos ( $\mathrm{P}>0,05)$. A média observada foi de $1,2 \%$ do peso corporal (PC) para a matéria seca, sendo inferior aos $2,2 \%$ PC encontrados por Gomes et al. (1994), em novilhas mestiças de aproximadamente $370 \mathrm{~kg}$ PC consumindo feno de capimcameroom com mais de $3,0 \mathrm{~m}$ de altura de corte no momento da fenação. $\mathrm{O}$ consumo foi uma das variáveis observadas e teve como objetivo avaliar o efeito do $\mathrm{S}$ na ingestão de forragens de baixo valor nutritivo. Kariuki et al. (1998) encontraram valores de $2,57 \%$ PC em consumo de capimnapier com 90 dias de idade.

O principal fator que pode ter afetado o consumo dos animais está relacionado aos teores de fibra em detergente neutro (FDN). O consumo obtido por Lopes \& Aroeira (1998), utilizando capim-elefante com idade de corte de 60 dias, foi de 1,3\% PC na FDN, tendo, no presente trabalho, sido encontrado $0,92 \%$ PC na FDN.

Pode-se observar um discreto aumento de consumo no tratamento $0,31 \%$, sendo possível, sugerir que esse tratamento acompanhou as recomendações da literatura.

O tratamento $0,92 \%$, proporcionou queda no consumo em comparação aos outros tratamentos e causou leve intoxicação em dois animais. Durante o ensaio, os animais apresentaram inapetência e falta de motilidade ruminal, características de intoxicação por enxofre, conforme apresentado por Preston \& Leng (1987). Estes autores sugerem que excesso de enxofre na dieta pode causar a redução do consumo.

Não foram observadas diferenças significativas entre os tratamentos para as contagens de protozoários ruminais $(\mathrm{P}>0,05)$, apresentadas na Figura 1. Os valores das contagens de protozoários apresentaram um pequeno aumento no tratamento $0,31 \%$, que teria o teor de enxofre de $3,1 \mathrm{~g}$ de $\mathrm{S} / \mathrm{kg}$ de MS, seria o teor ideal indicado pelo NRC (2001). Não foi observado nenhum efeito específico das doses de enxofre sobre estes grupos de protozoários, nem efeito nos animais. Em outras palavras, as populações de diferentes grupos de protozoários não variaram em função das diferentes doses de enxofre fornecidas.

TABELA 1 - Efeito dos períodos experimentais nos componentes nutritivos do capim-elefante, em base de matéria seca.

\begin{tabular}{ccccc}
\hline Períodos & MS & Fração (\%) \\
\cline { 2 - 5 } & 40,72 & FDN & FDA & PB \\
\hline $1^{\mathbf{0}}$ & 39,20 & 77,61 & 43,94 & 5,89 \\
$2^{\mathbf{o}}$ & 39,57 & 76,30 & 44,26 & 6,95 \\
$3^{\mathbf{0}}$ & 37,32 & 74,73 & 45,54 & 6,99 \\
$4^{\mathbf{0}}$ & 39,20 & 75,88 & 43,12 & 6,89 \\
Média & 1,4 & 76,13 & 44,22 & 0,8 \\
\hline DP & & 1,2 & 1,0 & \\
\hline
\end{tabular}

Ciênc. agrotec., Lavras, v. 31, n. 1, p. 218-222, jan./fev., 2007 
TABELA 2 - Consumo de matéria seca e FDN nos tratamentos.

\begin{tabular}{ccc}
\hline Tratamento & $\begin{array}{c}\text { Matéria Seca } \\
(\% \text { Peso corporal })\end{array}$ & $\begin{array}{c}\text { FDN } \\
(\% \text { Peso corporal })\end{array}$ \\
\hline $0,15 \%$ & 1,34 & 0,99 \\
$0,31 \%$ & 1,41 & 1,02 \\
$0,46 \%$ & 1,31 & 0,96 \\
$0,92 \%$ & 0,75 & 0,71 \\
\hline
\end{tabular}

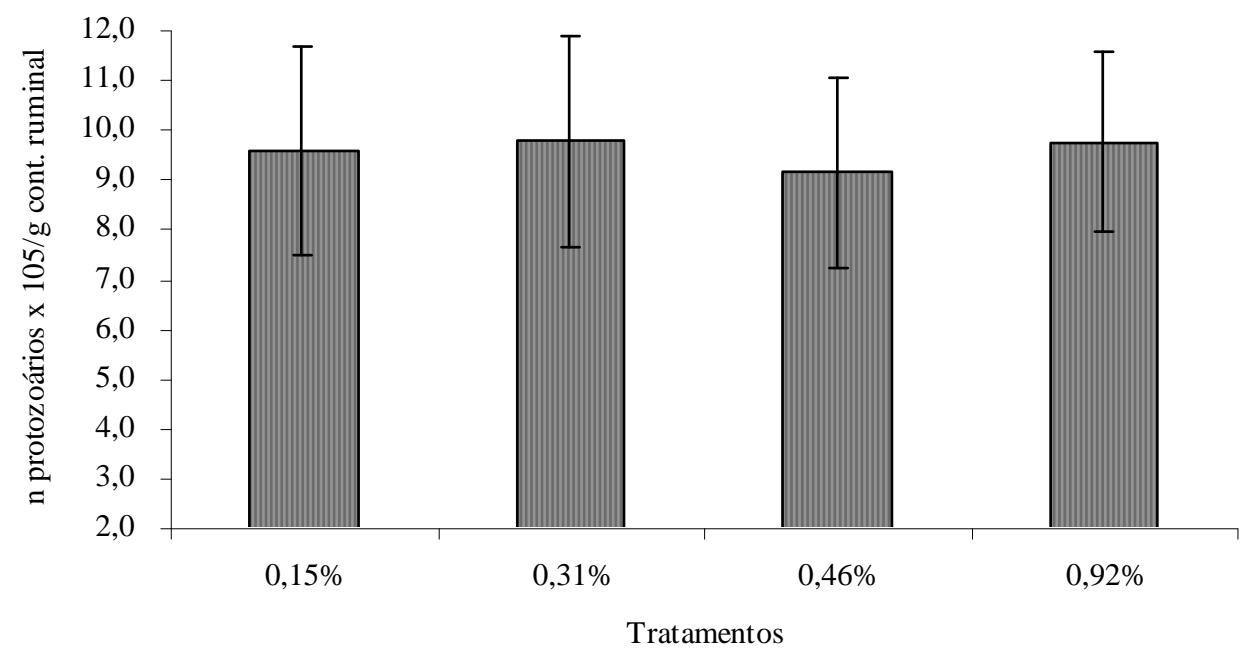

FIGURA 2 - Gráfico de médias de contagem de população de protozoários.

Esses resultados confirmam os encontrados por Morrison et al. (1990), que apresentaram aumento de bactérias, protozoários e fungos anaeróbios no rúmen, quando o conteúdo de enxofre da suplementação de feno de gramínea tropical aumenta de $0,04 \%$ para $0,07 \%$.

\section{CONCLUSÕES}

O consumo de matéria seca e FDN apresentaram tendência de superioridade no tratamento $0,31 \%$ de $\mathrm{S}$ na dieta, podendo ser recomendado como teor de enxofre na alimentação;

Teores de enxofre mais elevados do que os recomendados pela literatura podem levar a intoxicação;

\section{REFERÊNCIAS BIBLIOGRÁFICAS}

ANDRIGUETTO, J. M. Nutrição animal: as bases e os fundamentos da nutrição animal. 2. ed. São Paulo: [s.n.], 1993. v. 1.
ASSOCIATION OF OFFICIAL AGRICULTURAL CHEMISTS. Official methods of analysis. Washington, 1990. $1094 \mathrm{p}$.

D'AGOSTO, M.; CARNEIRO, M. E. Evaluation of lugol solution used for counting rumen ciliates. Revista Brasileira de Zootecnia, Viçosa, v. 16, n. 3, p. 725-729, 1999.

D’AGOSTO, M. et al. Comportamento e distribuição de protozoários ciliados (Protista, Ciliophora) no rúmen e no retículo de bovinos submetidos ao jejum. Revista Brasileira de Ciência e Veterinária, Belo Horizonte, v. 8, n. 1, p. 16-18, 2001.

DEHORITY, B. A. Laboratory manual for classification and morphology of rumen ciliate protozoa. Boca Raton: CRC, 1993. 325 p. 
EZEQUIEL, J. M. B. et al. Quantificação das bacterias sólidoaderidas, bactérias e protozoários líquidos-associados do rúmen de bovinos jovens alimentados com amiréia. Revista Brasileira de Zootecnia, Viçosa, v. 31, n. 2, p. 707-715, 2002.

GOMES, B. V. et al. Estudo das características físicosquímicas de fenos e palha e efeitos sobre ingestão, digestibilidade aparente e taxa de passagem da matéria seca, ph e concentração de amônia ruminal. Revista Brasileira de Zootecnia, Viçosa, v. 23, n. 3, p. 352-365, 1994.

KARIUKI, J. N. The potential of im proving napier grass under small holder dairy farmers' conditions in Kenya. 1998. 91 f. Thesis (Ph.D. in Animal Nutrition) - Wageningen Agricultural University, Wageningen, 1998.

LOPES, F. C. F.; AROEIRA, L. J. M. Consumo, digestibilidade e parâmetros ruminais em vacas Holandês $\mathrm{X}$ Zebu alimentadas com capim-elefante (Pennisetum purpureum Schum) picado. Arquivo Brasileiro de Medicina Veterinária e Zootecnia, Belo Horizonte, v. 50, n. 5, p. $593-$ 599, 1998.

MORRISON, M.; MURRAY, R. M.; BONIFACE, A. N. Nutrient metabolism and rumen microorganisms in sheep fed a poor-quality tropical grass hay supplemented with sulfate. Journal of Agricultural Science, Cambridge, v. 115, p. 269-275, 1990.

NATIONAL RESEARCH COUNCIL. Nutrient requirements of dairy cattle. 7 . ed. Washington: National Academy, 2001. 381 p.
OBISPO, N. E.; DEHORITY, B. A. Amost probable number method for enumeration of rumen fungi with studies on factors affecting their concentration in the rumen. Journal of Microbiological Methods, [S.1.], v. 16, p. 259-270, 1992.

PRESTON, T. R.; LENG R. A. Sulphur nutrition of ruminants. In: PRESTON, T. R.; LENG, R. A. (Eds.). Matching ruminant production systems with available resources in the tropics and sub-tropics. Armidale: Penambul Books, 1987. p. 46-47.

SILVA, D. J. da. Análise de alimentos: métodos químicos e biológicos. Viçosa: UFV, 1990. 166 p.

SISTEMAS PARA ANÁLISES ESTATÍSTICAS E GENÉTICAS. Manual de instruções. Versão 8.1. Viçosa: UFV, 2000. 142 p.

VIEIRA, R. A. M. et al. The influence of elephant-grass (Penniserum purpureum Schum., Mineiro variety) growth on the nutrient kinetics in the rumen. Animal Feed Science Technology, Amsterdam, v. 67, p. 151-161, 1997.

WESTON, R. H. et al. Feed intake and digestion responses in sheep to the addition of inorganic sulfur to a herbage diet of low sulfur content. Australian Journal of Agricultural Research, Victoria, v. 39, p. 1107-1119, 1988.

WILLIAMS, A. G. Rumen holotrich ciliate protozoa. Microbiology Reviews, [S.1.], v. 50, n. 1, p. 25-49, 1986.

WILLIAMS, A. G.; COLEMAN, G. S. The rumen protozoa. In: HOBSON, P. N.; STEWART, C. S. (Eds.). The rumen microbial ecosystem. London: Blackie Academic, 1997. v. 2, p. 73-139. 